\title{
Cheyletiella yasguri Smiley, 1965, un parasite de Canidés aux Etats-Unis et hyperparasite d'Hippoboscide en Iran (Acarina : Cbeyletidae)
}

\author{
par P.-H. VERCAMMEN-GRANDJEAN et H. RAK \\ (Travail de la G.W. Hooper Foundation, San Francisco, U.S.A.)
}

\begin{abstract}
Résumé
Cheyletiella yasguri Smiley, 1965, fut trouvé fort récemment parasitant des chiens en deux endroits de l'Etat de New York (U.S.A.). Plus récemment, il fut trouvé en hyperparasite phorétique sur un hippoboscide, lui-même parasite des chiens en Iran. Il est possible que C. yasguri ait été importé d'Asie Mineure aux EtatsUnis, par un quelconque voyageur canin. Le spécimen unique et femelle d'Iran est décrit et illustré intégralement et comparé avec un spécimen américain.
\end{abstract}

\section{Summary}

Cheyletiella yasguri Smiley, 1965, was found rather recently as a dog parasite, from 2 localities of the State of New York. More recently it was identified as a phoretic hyperparasite of a louse-fly. This one, Hippobosca longipennis, is itself a parasite from dogs in Iran and all Near East. Authors assume that $C$. yasguri might have been transported from the Near East to America by a traveling canine. The single species from Iran, a female, is completely described and illustrated, and compared with an American specimen.

\footnotetext{
* Le présent travail a été subventionné par les Services de Recherche de la Santé publique des Etats-Unis (Grant AI-03793) de l'Institut national pour l'Allergie et les Maladies infectieuses.

** Respectivement: 1) Parasitologiste de Recherche, G. W. Hooper Foundation, Université de Californie, Centre médical de San Francisco, California 94122, U.S.A. ; 2) Professeur Assistant, Département de Parasitologie, Faculté de Médecine Vétérinaire, Université de Téhéran (Iran), B.P. $\mathrm{n}^{\circ} 3262$.
} 
Cheyletiella yasguri fut décrit en 1965 par Robert L. Smiley lequel avait reçu des spécimens de ce Trombidiforme de deux endroits différents de l'Etat de New-York. Ils avaient été récoltés, en 1962 et 1963 , sur des chiens qui, selon les dires d'un des découvreurs, le médecin vétérinaire I. Yasgur, semblaient en souffrir (4). Entre temps, en mai 1966, l'un d'entre nous (H. R.) découvrait un petit acarien rouge attaché au cou d'un hippobosque capturé à Téhéran. Ce dernier, Hippobosca longipennis Fabricius, 1805, est le seul connu dans le monde comme étant parasite de Mammifères carnivores, dont les canidés* (2). Par ailleurs, l'unique exemplaire (une femelle) recueillie en Iran fut identifiée grâce à la bienveillance de notre collègue Smiley qui voulut bien nous prêter son précieux matériel pour comparaison. Il s'agissait bien de Cheyletiella yasguri Smiley, 1965 et les spécimens étaient même étonnamment identiques, ainsi que le montrent d'ailleurs les mesures (p. 3).

Cette identité semble témoigner d'une communauté d'origine récente, mais quel est le véritable berceau de $C$. yasguri, l'Amérique ou l'Asie Mineure ? C'est bien difficile à déterminer avec certitude. Toutefois, il semble plus plausible que ce soit l'Asie Mineure et que l'importation d'un chien infesté puisse être à l'origine de sa très récente découverte aux Etats-Unis. Qu'un Américain se donne la peine de transporter un Chien du Proche-Orient aux U.S.A. est très concevable, et l'inverse semble devoir être plus rare. Par ailleurs, connaissant la nature attentive des Américains vis-à-vis de leurs compagnons favoris, les chiens, il est bien étonnant que l'existence de cet ennuyeux parasite n'ait verte aux Etats-Unis. Qu'un Américain se donne la peine de transporter un chien du pas été signalée d'Amérique avant 1965. Par contre, le Chien ne jouit pas en Asie de la même sollicitude que son congénère américain et c'est sans doute pourquoi ses microparasites - dont $C$. yasguri - ne furent jamais identifiés proprement dans cette partie du monde. Enfin l'argument de poids est celui de la phorésie pratiquée par $C$. yasguri sur l'hippobosque du chien; phorésie qui semble témoigner d'une pratique courante et ancienne du fait de la parfaite acceptation, par la mouche parasite, de son hyperparasite acarien **.

Vu l'intérêt multiple de Cheyletiella yasguri, nous jugeons opportun de redonner une description détaillée de sa femelle, accompagnée d'une iconographie intégrale.

Nous tenons à remercier ici, les collègues Edward W. Baker et Robert L. Smiley, tous deux de l'Entomology Research Division A.R.S., U.S. Departement of Agriculture, Washington D.C., pour nous avoir fourni de précieuses informations et nous avoir permis de voir le matériel américain de $C$. yasguri. Egalement M. le Pr J.-C. Béquaert, de l'Université d'Arizona à Tucson, ainsi que M. le $\mathrm{D}^{\mathrm{r}}$ Tsing C. Maa du Musée Bernice-

* Hippobosca longipennis Fabricius, 1805 possède 9 synonymes: 1) H. capensis Olfers, 1816 (S. Afr., Cape); 2) H. francilloni Leach, 1817 (India); 3) H. orientalis Macquart, 1843 (India); 4) H. laticornis Macquart, 1843 ; 5) H. fossulata Macquart, 1843 (S. Amer.) ; 6) Ornithomyia chinensis Giglioli, 1864 (Fukien) ; 7) H. canina Rondani, 1878 (Europe, Asie Mineure, Afrique) ; 8) H. cunicosa Thalhammer, 1899 (Hongrie); et 9) H. equina v. canina Dryensky, 1926 (Bulgarie).

** Il y a peu, le $\mathrm{D}^{\mathrm{r}}$ Michel Lavoipierre, Professeur à 1'Université de Californie (Davis Campus), me fit part de ce qu'il avait récolté à son tour, sur des chiens californiens, des Acariens qu'il identifia à $C$. yasguri. Il me prêta aimablement son matériel que j'ai reconnu également comme $C$. yasguri.

M. Lavoipierre estime que bon nombre de cas d'espèce furent erronément attribués à $C$. parasitivorax dans le passé, qui n'étaient autres que dus à $C$. yasguri. 
Bishop à Honolulu (Hawaï), pour leurs aimables renseignements et identifications en matière d'Hippoboscides. Notre reconnaissance va aussi à $\mathrm{M}$. le $\mathrm{P}^{\mathrm{r}} \mathrm{A}$. Rafyi, Doyen de la Faculté Vétérinaire de l'Université de Téhéran, qui a bien voulu patronner notre association d'étude.

\section{Cbeyletiella yasguri smiley, 1965.}

\section{A. - Description.}

1) Mesures : exprimées en microns, du type femelle d'Iran (I) et seul spécimen, comparées à celles d'un spécimen paratype femelle d'Amérique (A), n 2956-PT, récolté par I. Yasgur sur chien («Schnauzer pups») à Mamaroneck (State of New York) en 1963.

\begin{tabular}{|c|c|c|c|c|c|c|c|c|c|c|c|c|c|}
\hline & & DIOSOM & & & SCUTL & & & & & LSITÉ & IDIOSOMA & & \\
\hline & & ongueu & & & arg. : & & ong. : & Pré & é-scutau & & Scutoux : & $\mathrm{Hu}$ & uméraux : \\
\hline & Prop. & Hyst. & Total & Anter. & Post. & & Tot. & Anter. & Med. & Post. & (4 nus) & Ante & er. Post. \\
\hline I & 182 & 220 & 402 & 132 & 300 & & 152 & 50 & 92 & 106 & 84 & 130 & 116 \\
\hline A & 174 & 222 & 396 & 128 & 273 & & 145 & 44 & 81 & 104 & 82 & 145 & 125 \\
\hline & & Largeu & & & Scute & ULUM & & Pré-sc & cutulaux & : Post & t-scutulaı & $u x: 1$ & Sternaux \\
\hline & Prop. & Hyst. & Pygos. & Lon: & & Lar & g. : & Barbe & $\mathrm{N}$ & $\mathrm{Nu}$ & Barbe & & (3 nus) \\
\hline I & 304 & 270 & 176 & 54 & & 3 & & 180 & 96 & 44 & 70 & & $40-44$ \\
\hline A & 300 & 276 & 184 & 45 & & 3 & & 169 & 92 & - & - & & - \\
\hline & & Hauteu & & & PATTI & ES (1 & Long. & & PIL & $\begin{array}{r}\text { SITÉ PY } \\
\text { (nu }\end{array}$ & $\begin{array}{l}\text { YGosom } \\
\text { le) }\end{array}$ & & $\begin{array}{l}\text { UROPOR. } \\
\text { P. }\end{array}$ \\
\hline & Prop. & Hyst. & Constr & $P_{1}$ & $\mathbf{P}_{2}$ & $P_{3}$ & $\mathrm{P}_{4}$ & IP & Ext. & $\begin{array}{l}\text { Pré- } \\
\text { ext. }\end{array}$ & $\begin{array}{r}\text { Péri-vu } \\
\text { (14) }\end{array}$ & & (6 nus) \\
\hline I & 180 & 190 & 130 & 270 & 250 & 260 & 340 & 1120 & 324 & 48 & $22-$ & & $26-32$ \\
\hline A & 165 & 180 & 130 & 252 & 2.24 & 252 & 322 & 1050 & - & - & - & & - \\
\hline
\end{tabular}

Abréviations :

Prop. $=$ Propodosome $;$ Hyst. $=$ Hystérosome $;$ Pygos. $=$ Pygosome $;$ Scutum $=$ écusson $;$

Constr. $=$ Constriction entre Propodosome et Hystérosome; Anter. = Antérieur ;

Méd. $=$ Médian $;$ Post. $=$ Postérieur $;$ Scutulum $=$ petit écusson sclérifié, deux sur l'hystérosome $; \mathrm{IP}=$ Index Pedibus $=$ somme des longueurs des pattes $\left(\mathrm{P}_{1}+\mathrm{P}_{2}+\mathrm{P}_{3}+\right.$ $\mathrm{P}_{4}$ ), donne, en un seul nombre, une idée relative de la taille d'un acarien ; surtout précieux lorsque l'idiosome se modifie par engorgement.

Voir texte pour d'autres significations complémentaires. 
Cheyletiella yasguri Smiley, 1965

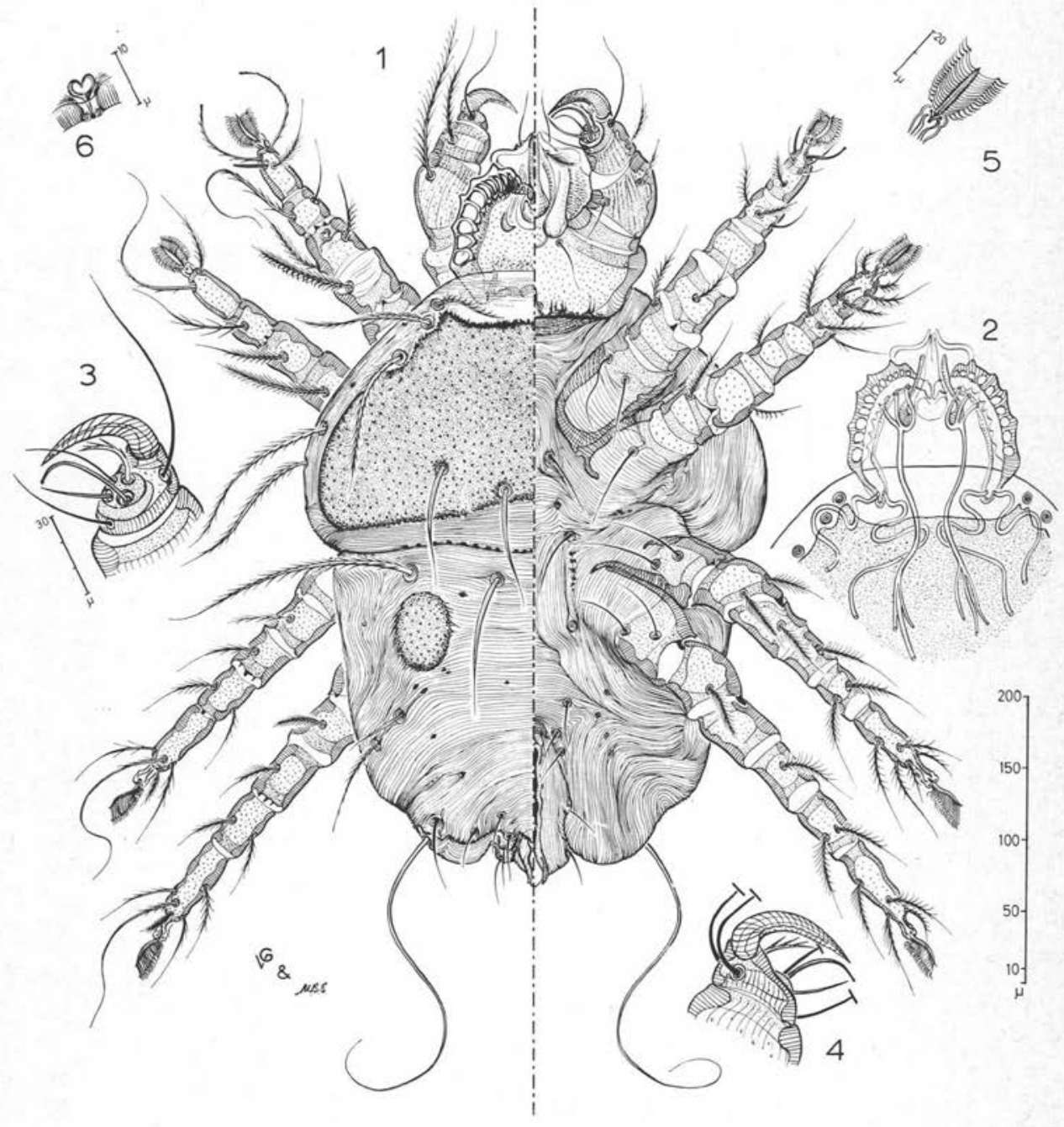


2) Idiosome : (fig. 1) en forme de gland, duquel la cupule est le propodosome, et dont le tégument externe est profondément piissé, exception faite du scutum ou écusson, les deux scutula (du latin scutulum = petit écusson) ou écussons hystérosomaux, des coxae (souvent partiellement plissés) et de ia gnathobase. Le «scutum » ou écusson antéro-dorsal est sub-trapézoidal et perforé de nombreux canalicules poreux, bien marqués et assez disséminés. Les trois paires de poils barbelés antérieurs se trouvent en bordure de l'écusson (six pré-scutaux: deux antérieurs, deux médians et deux postérieurs). Deux paires de poils barbelés huméraux sont également extrascutaux. Tandis que deux paires de poils forts et nus, sont subcentraux dans l'aire postérieure du scutum. Il n'y a pas d'yeux. L'hystérosome est bien séparé du propodosome par une constriction marquée. Deux peiits écussons vestigiels (scutulum) rappellent le large écusson hystérosomal du mâle; ils sont ponctués comme l'écusson. Deux paires de poils préscutulaires: deux externes longs et barbelés $(180 \mu)$, deux internes nus et semblables aux deux paires de scutaux $(96 \mu)$. Des deux paires de poils post-scutulaires, la paire antéro-interne est courte et nue $(44 \mu)$, tandis que la postéro-externe est barbelée $(70 \mu)$. La région pygosomale est bien définie et large de $170 \mathrm{u}$; elle incorpore les périphéries vulvaires et uroporales. Aux limites externes du pygosome, deux paires de poils nus, la plus externe très longue et épaisse à sa base $(324 \mu)$, l'autre plus courte $(48 \mu)$. Les soies péri-vulvaires, au nombre de sept paires, ont des dimensions variant entre 22 et $32 \mu$. Le soies péri-uroporales sont au nombre de trois paires et varient entre 26 et $32 \mu$.

3) Pattes : (figs. 1, 5 et 6) toutes quatre paires, sont constituées de six articles bien sclérifiés et croissant en diamètre du tarse au coxa. L'espace intercoxal ou sternal porte trois paires de soies nues, $\mathrm{fSt}=2 \mathrm{~N} .2 \mathrm{~N} .2 \mathrm{~N}$. Les coxae sont ponctués, mais en grande partie couverts de plissements épiostracaux ; la seconde paire est garnie d'une seule paire de soies nues, les trois autres en possèdent chacune deux et $f C x=2 N . N .2 N$. $2 \mathrm{~N}$. Trochanters arrondis et garnis tous d'au-moins un poil barbelé : $\mathrm{fTr}=\mathrm{B} \cdot \mathrm{B} .2 \mathrm{~B} . \mathrm{B}$. Fémurs avec un ou deux poils barbelés: $\mathrm{fF}=2 \mathrm{~B} .2 \mathrm{~B} .2 \mathrm{~B}$.B. Le genu antérieur est le seul à posséder un organe particulier, sous forme d'un petit solenidion cardiforme au pédoncule profondément serti dans une invagination antéro-dorsale (fig. 6) ; hormis cet organe particulier, le $\mathrm{fG}=2 \mathrm{~B} \cdot 2 \mathrm{~B} \cdot 2 \mathrm{~B} .2 \mathrm{~B}$. Les tibias des pattes 3 et 4 ont quatre poils barbelés subégaux, le tibia 2 en a quatre également mais le dorsal est long et particulier, quant au tibia 1 il possède deux poils barbelés subégaux et ventraux, deux soies nues (tibiala) et, en gros, on a un $\mathrm{fTi}=3 \mathrm{~B} \cdot 4 \mathrm{~B} \cdot 4 \mathrm{~B} .4 \mathrm{~B}$. Le tarse 1 possède un solenidion dorsal, deux poils courbes à barbes courtes et à extrémités obtuses, deux solenidions ventraux dont l'un à extrémité également obtuse et trois soies fines, plus deux poils barbelés ordinaires; le tarse 2 a un solenidion ventral et une soie (tarsala), plus six poils barbelés, dont un dorso-apical est un fouet garni de quelques barbes courtes; les tarses 3 et 4 possèdent chacun neuf poils barbelés, dont un dorso-apical en forme de fouet garni de quelques barbes courtes, et fTa $=4$ B.6B.9B.9B.

Le petit prétarse de chaque patte porte un empodium lancéolé. et pectiné, sorte de rachis gladéolé garni de dix-sept paires de barbes griffues (fig. 5) (voit table, p. 6). 


\begin{tabular}{|c|c|c|c|c|c|c|c|c|c|c|c|c|c|c|c|}
\hline \multirow{3}{*}{$\begin{array}{l}\text { TABles } \\
\text { Article }\end{array}$} & \multicolumn{12}{|c|}{ PATtes } & \multirow{2}{*}{\multicolumn{3}{|c|}{$\begin{array}{c}\text { PALPE } \\
L=200 \mu\end{array}$}} \\
\hline & \multicolumn{3}{|c|}{$P_{1}=264 \mu$} & \multicolumn{3}{|c|}{$P_{2}=252 \mu$} & \multicolumn{3}{|c|}{$\mathrm{P}_{3}=255 \mu$} & \multicolumn{3}{|c|}{$P_{4}=328 \mu$} & & & \\
\hline & $\mathrm{N}$ & nature & $\mu$ & $\mathrm{N}$ & nature & $\mu$ & $\mathbf{N}$ & nature & $\mu$ & $\mathrm{N}$ & nature & $\mu$ & $\mathrm{N}$ & nature & $\mu$ \\
\hline \multirow[t]{2}{*}{$\begin{array}{c}\text { CoXa OU } \\
\text { GNATHOBASE : }\end{array}$} & & $\begin{array}{l}\text { larg. } \\
\text { long. }\end{array}$ & $\begin{array}{l}50 \\
84 \\
\end{array}$ & & $\begin{array}{l}\text { larg. } \\
\text { long. }\end{array}$ & $\begin{array}{l}44 \\
66 \\
\end{array}$ & & $\begin{array}{l}\text { larg. } \\
\text { long. }\end{array}$ & $\begin{array}{l}46 \\
50 \\
\end{array}$ & & $\begin{array}{l}\text { larg. } \\
\text { long. }\end{array}$ & $\begin{array}{l}52 \\
80\end{array}$ & & $\begin{array}{l}\text { larg. } \\
\text { long. }\end{array}$ & $\begin{array}{r}156 \\
60 \\
\end{array}$ \\
\hline & 2 & $\mathrm{~N}$ & $\overline{54}$ & 1 & $\mathrm{~N}$ & 56 & 2 & $\mathrm{~N}$ & 56 & 2 & $\mathbf{N}$ & 44 & 2 & $\mathrm{~N}$ & 36 \\
\hline \multirow[t]{2}{*}{ TROCHANTER : } & & $\begin{array}{c}\text { long. } \\
-\end{array}$ & 32 & & long. & 34 & . & long. & 40 & & $\begin{array}{c}\text { long. } \\
-\end{array}$ & 50 & & $\begin{array}{l}\text { long. } \\
\text { larg. }\end{array}$ & $\begin{array}{l}15 \\
48 \\
\end{array}$ \\
\hline & 1 & B (v) & 56 & $\overline{1}$ & B (v) & 56 & 2 & B (v) & 48 & 1 & B (v) & 48 & & - & \\
\hline \multirow[t]{3}{*}{ FÉMUR : } & & long. & 40 & & long. & 40 & & long. & 36 & & long. & 40 & & $\begin{array}{l}\text { long. } \\
\text { larg. }\end{array}$ & $\begin{array}{l}50 \\
50 \\
\end{array}$ \\
\hline & 1 & B (d) & $\overline{76}$ & 1 & B (d) & 84 & $\overline{1}$ & B (d) & $\overline{44}$ & 1 & B (d) & 36 & 1 & B (d) & $\overline{112}$ \\
\hline & 1 & B (v) & 42 & 1 & B (v) & 36 & 1 & B (v) & 48 & & - & & $\begin{array}{l}1 \\
1\end{array}$ & $\begin{array}{l}B(v) \\
N(v)\end{array}$ & $\begin{array}{l}38 \\
30 \\
\end{array}$ \\
\hline \multirow[t]{4}{*}{ GENU : } & 1 & $\begin{array}{l}\text { long. } \\
\text { S/card. }\end{array}$ & $\begin{array}{r}34 \\
7 \\
\end{array}$ & & $\begin{array}{c}\text { long. } \\
- \\
\end{array}$ & 36 & & $\begin{array}{c}\text { long. } \\
-\end{array}$ & 30 & & long. & 40 & & $\begin{array}{l}\text { long. } \\
\text { larg. }\end{array}$ & $\begin{array}{l}18 \\
32 \\
\end{array}$ \\
\hline & & & 4 & & & & & & & & & & & & \\
\hline & 1 & B (d) & 64 & 1 & B (d) & 62 & 1 & B (d) & 60 & 1 & B (d) & 46 & 1 & B (d) & 82 \\
\hline & 1 & B (d) & 40 & 1 & B (v) & 36 & 1 & B (v) & 64 & 1 & B (v) & 60 & 1 & B (v) & 48 \\
\hline \multirow[t]{6}{*}{ TIBIA : } & & long. & 30 & & long. & 30 & & long. & 34 & & long. & 44 & & $\begin{array}{l}\text { long. } \\
\text { larg. }\end{array}$ & $\begin{array}{l}30 \\
30 \\
\end{array}$ \\
\hline & 1 & $N(d)$ & 22 & & - & & & - & & & - & & & (d) & 68 \\
\hline & 1 & B (d) & 62 & 1 & B (d) & 86 & 2 & B (d) & 52 & 2 & B (d) & 44 & 3 & $N\{(1)$ & 64 \\
\hline & 1 & $N(v)$ & 30 & & - & & & - & & & - & & & (v) & 40 \\
\hline & 2 & B (v) & $\overline{(30}$ & 2 & B (v) & 66 & 2 & B (v) & 62 & 2 & B (v) & 60 & 1 & Griffe & 50 \\
\hline & & & 44 & 1 & B (l) & 40 & & & & & & & & simple & 10 \\
\hline \multirow[t]{7}{*}{ TARSE : } & & long. & 44 & & long. & 42 & . & long. & 65 & & long. & 74 & & long. & 12 \\
\hline & 2 & B (ad) & $\left(\begin{array}{l}70 \\
78\end{array}\right.$ & 1 & B (ad) & 136 & 1 & B (ad) & 136 & 1 & B (ad) & 114 & & diam. & 15 \\
\hline & 1 & $S$ (d) & $\overline{24}$ & 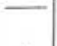 & - & & 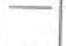 & - & & & - & & 1 & $\mathrm{~S}$ & 9 \\
\hline & 1 & B (d) & 52 & 1 & $B$ (d) & 60 & 2 & $B$ (d) & 64 & 3 & B (d) & 54 & 1 & B & 7 \\
\hline & 2 & $\begin{array}{r}\text { Term. } \\
\text { (v) }\end{array}$ & $\left(\begin{array}{l}30 \\
20\end{array}\right.$ & & - & & & - & & & - & & 1 & Term. & 6 \\
\hline & 3 & $N(v)$ & 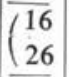 & 1 & $N(v)$ & 15 & & - & & & - & & 1 & $\mathrm{~N}$ & 8 \\
\hline & 1 & B (v) & $\overline{27}$ & 4 & B (v) & $\left(\begin{array}{l}24 \\
48\end{array}\right)$ & 5 & B (v) & $\left(\begin{array}{l}30 \\
60\end{array}\right)$ & 4 & B (v) & $\left(\begin{array}{l}28 \\
09\end{array}\right)$ & & - & \\
\hline
\end{tabular}


4) Gnathosome : (figures 1, 2, 3 et 4) puissant et bien armé, attaché à l'idiosome par une gnathobase large mais courte, bien sclérifiée. Palpes solides et trapues, avec fémur et genu strio-ponctué et tibia très court; gnathobase avec deux soies nues; court trochanter, sans poil ; long fémur avec une soie nue et un court poil barbelé à la face externe (ventrale sur le dessin) et un long barbelé à la face dorsale ; genu plus court avec un poil barbelé et court à la face externe et un plus long barbelé à la face dorsale ; tibia très court avec trois soies nues, une ventrale courte, une externe et une dorsale plus longues, griffe tibiale forte et rainurée ; tarse plat et court avec un fort solenidion (apex obtus), une soie nue (tarsala), une fine soie nue apicale et un poil barbelé (deux ou trois courtes barbes) (figures 3 et 4). Chélicères ramenées aux proportions de simples baguettes internes, sans fonction apparente de fixation: galea en forme d'as de pique engainant les deux chélostyles et portant une paire de soies nues apicales (galeala $=40 \mu$ ) et une autre paire de poils barbelés ventraux et subterminaux $(26 \mu)$.

Péritrèmes (fig. 1) et système trachéal (fig. 2) ; péritrèmes en forme de $\mathrm{M}$ avec cinq crêtes latéro-externes et environ dix à quatorze spiracles polygonaux de chaque côté. Ces derniers s'ouvrent dans des alvéoles sphériques, lesquels communiquent avec une chambre à air allongée dont partent au moins cinq paires de tracheés. (Voir p. 6, la table du palpe).

\section{B. - Hôte et Parasitope.}

1) Sur le Chien, à la base des poils (Teigne).

2) Sur Hippobosque, accroché au cou.

\section{c. - Localités et Dates.}

1) Sur Chien aux Etats-Unis («Schnauzer pups ») à Mamaroneck (New York) en avril 1963, et également de Chiens de l'Université Cornell à Ithaca (N. Y.) en 1962.

2) Sur Hippobosca longipennis Fabricius, 1805, capturé à Téhéran (Iran) le 11 mai 1966.

\section{D. - Matériel Type.}

1) Femelle holotype : U.S. Nat. Mus. $\mathrm{n}^{\circ} 2956$ et un paratype (vu par nous), provenant de la série de Mamaroneck fournie par le $\mathrm{D}^{r} \mathrm{I}$. Yasgur.

2) Femelle type $\mathrm{n}^{\circ} 11566 / 1$ en provenance d'Iran ; a été déposée également avec les types américains trouvés sur Chien, au U.S. Nat. Muséum de Washington.

\section{Bibliographie}

1. BAKER (E. W.), 1949. - A Review of the mites of the Family Cheyletidae in the United States National Museum., Proc. U.S. Nat. Mus., Washington, 99, 267-320. 
2. BÉQuaert (J.C.), 1953. - The Hippoboscidae or Louse-flies (Diptera) of Mammals and Birds. Part I. Structure, Physiology and Natural History., Entom. Amer., 32 et 33, 1-209 et 211-442.

—, 1954 à 1957. - The Hippoboscidae or Louse-flies (Diptera) of Mammals and Birds. Part. II. Taxonomy, Evolution and Revision of American genera and species., Entom. Amer., 34, 35 et 35, 3-181, 183-415 et 417-611.

3. Lawrence (R. F.). 1956. - Studies on South Africa fur mites., Ann. Natal Mus., 13, 337-374.

4. Smiley (R. L.), 1965. - Two new species of the genus Cheyletiella (Acarina: Cheyletidae). Proc. Entom. Soc. Washington, 67, 75-79.

5. Volgin (V. I.), 1960. - On the taxonomy of predatory mites of the family Cheyletidae. II. Genus Cheyletiella Can., Akad. Nauk, Zool. Inst. Otdel. Parasitol. Sbornikn., $19,237-248$.

\section{NOTES ET INFORMATIONS}

$4^{\circ}$ Conférence Internationale de Parasitologie Vétérinaire: Cette Conférence intitulée «Pathologie des maladies parasitaires» (Pathology of Parasitic Diseases) se tiendra à l'Ecole vétérinaire de l'Université de Glasgow (Ecosse) du 26 au 28 août 1969. Envoi des titres des communications avant le $1^{\text {er }}$ février 1969 au Secrétaire-Trésorier, le Dr. S. M. Gaafar, Dept. of Veterinary Microbiology, Purdue University, Lafayette Indiana 47905, U.S.A., ou au Dr. E. S. L. Soulsly, Dept. of Pathobiology, School of Veterinary Medicine, University of Pennsylvania, Philadelphia, Pennsylvania 19104, U.S.A. 